\title{
THE CONNECTION MAP FOR ATTRACTOR-REPELLER PAIRS
}

\author{
CHRISTOPHER MCCORD
}

\begin{abstract}
In the Conley index theory, the connection map of the homology attractor-repeller sequence provides a means of detecting connecting orbits between a repeller and attractor in an isolated invariant set. In this work, the connection map is shown to be additive: under suitable decompositions of the connecting orbit set, the connection map of the invariant set equals the sum of the connection maps of the decomposition elements. This refines the information provided by the homology attractor-repeller sequence. In particular, the properties of the connection map lead to a characterization of isolated invariant sets with hyperbolic critical points as an attractor-repeller pair.
\end{abstract}

1. The homology attractor-repeller sequence. One of the methods by which the Conley index theory studies isolated invariant sets is to decompose them into subinvariant sets and connecting orbits between the sets. The simplest such decomposition of a set $S$ is an attractor-repeller pair $\left(A, A^{*}\right)$ : an invariant subset $A$ of $S$ with $A=\omega(U)$ for some $S$-neighborhood $U$ of $A$; and its dual repeller $A^{*}=\{x \in S \mid \omega(x) \cap A=\varnothing\}$. Then $A^{*}$ is a repeller in $S$, and $S$ decomposes into $A \cup C\left(A^{*}, A ; S\right) \cup A^{*}$, where

$$
C\left(A^{*}, A ; S\right)=\left\{x \in S \mid \omega^{*}(x) \subseteq A^{*}, \omega(x) \subseteq A\right\}
$$

is the connecting orbit set.

If $S$ is an isolated invariant set with attractor-repeller pair $\left(A, A^{*}\right)$, then $A$ and $A^{*}$ are also isolated, so $S, A$ and $A^{*}$ all have a Conley index in $X$, defined in terms of index pairs for each in $X$. These index pairs are related by the construction of an index triple: a compact triple $N_{0} \subseteq N_{1} \subseteq N_{2}$ such that $\left(N_{2}, N_{0}\right)$ is an index pair for $S$ in $X ;\left(N_{1}, N_{0}\right)$ is an index pair for $A$ in $X ;\left(N_{2}, N_{1}\right)$ is an index pair for $A^{*}$ in $X$. Kurland [4] shows that index triples exist for all $\left(S ; A, A^{*}\right)$. Further, these index triples can always be taken to be regular $[\mathbf{7}, \mathbf{8}]$ (i.e. each index pair induced by the triple is an NDR pair).

From the index triple of $\left(S ; A, A^{*}\right)$, Kurland constructs a long coexact sequence relating the (homotopy) Conley indices of $S, A$, and $A^{*}$ in $X$. Franzosa [3] derives from this an exact sequence relating the homology Conley indices. Namely, given a regular index triple $\left(N_{2}, N_{1}, N_{0}\right)$ for an attractor-repeller pair $\left(A, A^{*}\right)$ in $S$, there exists an exact sequence

$$
\cdots \stackrel{\partial}{\rightarrow} H_{*}\left(N_{1}, N_{0}\right) \stackrel{i .}{\rightarrow} H_{*}\left(N_{2}, N_{0}\right) \stackrel{j_{*}}{\rightarrow} H_{*}\left(N_{2}, N_{1}\right) \stackrel{\partial}{\rightarrow} \cdots .
$$

Received by the editors January 12, 1987 and, in revised form, March 20, 1987.

1980 Mathematics Subject Classification (1985 Revision). Primary 34C35; Secondary 34C40, $58 \mathrm{~F} 12$.

Key words and phrases. Conley index, attractor-repeller pair, connection map. 
This defines an exact sequence of homology Conley indices

$$
\cdots \stackrel{\partial}{\rightarrow} C H_{*}(X ; A) \stackrel{i_{*}}{\longrightarrow} C H_{*}(X ; S) \stackrel{j_{*}}{\longrightarrow} C H_{*}\left(X ; A^{*}\right) \stackrel{\partial}{\rightarrow} \cdots
$$

called the homology index sequence of the attractor-repeller pair, or the homology attractor-repeller sequence. This sequence provides an algebraic condition for the existence of connections. Namely, if the connection map $\partial$ is nontrivial, then $C\left(A^{*}, A ; S\right)$ is nonempty. In $[2, \mathbf{3}]$ Franzosa generalizes the connection map for an attractor-repeller pair to a connection matrix for a Morse decomposition. Connection maps and matrices are used by many authors (e.g. $[\mathbf{1 - 4}, \mathbf{7}-\mathbf{1 0}])$ to detect and (when combined with other techniques) describe connecting orbit sets in a variety of problems.

It is not true that $\partial$ is nontrivial when $C\left(A^{*}, A ; S\right)$ is nontrivial. For example, consider the gravity flow on the $k$-sphere $S^{k}(k>0)$. This gives a gradient flow with the south pole $x_{0}$ an attractor; the north pole $x_{1}$ a repeller; and $C\left(x_{1}, x_{0} ; S^{k}\right)$ is nonempty. Further, $C H_{*}\left(S^{k} ; S^{k}\right) \cong H_{*}\left(S^{k}\right) ; C H_{*}\left(S^{k} ; x_{1}\right) \cong \tilde{H}_{*}\left(S^{k}\right) ;$ and $C H_{*}\left(S^{k} ; x_{0}\right) \cong \tilde{H}_{*}\left(S^{0}\right)$. That is, for all $k$,

$$
C H_{*}\left(S^{k} ; S^{k}\right) \cong C H_{*}\left(S^{k} ; x_{1}\right) \oplus C H_{*}\left(S^{k} ; x_{0}\right),
$$

and $\partial$ is trivial. For $k>1$, this is simply the fact that the dimensions of the attractor and repeller indices do not admit a nontrivial degree 1 map. (If dimensions admit a nontrivial degree 0 map, the transition matrix of [10] provides information similar to that of the connection map.) However, when $k=1, C\left(x_{1}, x_{0} ; S^{k}\right)$ consists of two orbits and the dimensions admit a nontrivial map

$$
\partial: C H_{1}\left(S^{k} ; x_{1}\right) \rightarrow C H_{0}\left(S^{k} ; x_{0}\right),
$$

yet $\partial$ is trivial.

This triviality can be understood by considering each connecting orbit separately. The closure of each orbit is isolated, with $\left(x_{1}, x_{0}\right)$ an attractor-repeller pair and the connection map of the orbit an isomorphism. However, these isomorphisms are of opposite orientation, so their sum is trivial. That is, the sum of the connection maps of the individual orbits gives the connection map of the circle.

In this work we formalize and generalize this additivity by producing a sum theorem for the connection map which holds for any attractor-repeller pair $\left(A, A^{*}\right)$ of an isolated invariant set $S$. This requires identifying isolated subsets of $S$ which each have $\left(A, A^{*}\right)$ as an attractor-repeller pair and whose union is $S$. In $\S 2$, conditions for such decompositions are found (Theorem 2.2) and the sum theorem is proved for such decompositions (Theorem 2.5). This provides new methods of detecting connecting orbits (2.6-3.3), but it also can be reapplied to the flows on spheres which motivated it, refining the information available there. Namely, it is shown (Theorem 3.4) that the $k$-sphere remains isolated whenever it is embedded into a $C^{2}$ flow on a manifold so that $x_{1}$ and $x_{0}$ remain hyperbolic with indices $k$ and 0 , and (with one exception when $k=1$ ) it is the only isolated set which can be so embedded.

The reader is referred to $[\mathbf{1}, \mathbf{1 1}]$ for attractor-repeller pairs and index constructions, and to [3] for connection maps. The notation in this paper follows that of [6]: IS denotes the category of pairs $(X ; S)$ with $X$ a locally compact metric flow and $S \subseteq X$ an isolated invariant set; $\mathscr{N}(X ; S)$ denotes the collection of index pairs 
$(N, L)$ for $S$ in $X$ such that $L$ is the "immediate exit set" of $N$, and such that $N$ with the "immediate entrance set" $L^{-}$is an index pair for the reverse flow; $C H_{*}(X ; S)$ is the homology Conley index of $S$ in $X$, defined by $C H_{*}(X ; S) \cong H_{*}(N, L)$ for any $(N, L) \in \mathscr{N}(X ; S)$.

I would like to thank Henry Kurland for pointing out counterexamples to the converse of Lemma 2.1. The examples, and an alternate proof of Lemma 2.1, can be found in $[\mathbf{5}]$.

2. The sum theorem for attractor-repeller pairs. To develop the sum theorem, the appropriate method of decomposing an attractor-repeller decomposition will be to take separations of the connecting orbit set. Recall that if $\mathscr{A}$ is an indexing set, $X$ a topological space, an $\mathscr{A}$-separation of $X$ is a collection $\left\{X_{\alpha} \mid \alpha \in \mathscr{A}\right\}$ of disjoint open subsets of $X$ such that $X=\bigcup_{\alpha \in \mathscr{A}} X_{\alpha}$. Note that $U_{\alpha}=\bigcap_{\beta \neq \alpha}\left(X \backslash U_{\beta}\right)$ is also closed in $X$. For a space $X$ with a flow defined on it, an invariant $\mathscr{A}$-separation will refer to a separation with each $X_{\alpha}$ invariant under the flow. If $S$ is a compact invariant set with $\left\{S_{\alpha}\right\}_{\alpha \in \mathscr{A}}$ an invariant $\mathscr{A}$-separation of $S$, then $\mathscr{A}$ is finite. Similarly, if $\left(A, A^{*}\right)$ is an attractor-repeller pair for $S$ with $\left\{C_{\beta}\right\}_{\beta \in \mathscr{B}}$ an invariant $\mathscr{B}$-separation of $C\left(A^{*}, A ; S\right)$, then $\mathscr{B}$ is finite. Each such $C_{i}$ is then a union of components of $C\left(A^{*}, A ; S\right)$, so $C\left(A^{*}, A ; S\right)$ will have a finest separation if and only if it has a finite number of components.

LEMMA 2.1. Suppose $S$ is a compact invariant set in $X$ with attractor-repeller pair $\left(A, A^{*}\right)$. If $A^{*}$ and $A$ are isolated in $X$ and $C\left(A^{*}, A ; S\right)$ is open in $C\left(A^{*}, A ; X\right)$, then $S$ is isolated in $X$.

Proof. Choose disjoint index pairs $\left(N_{1}, L_{1}\right) \in \mathcal{N}\left(X ; A^{*}\right),\left(N_{0}, L_{0}\right) \in \mathcal{N}(X ; A)$. There exist $T_{1}<0<T_{0}$ such that $S \cap L_{1}=C\left(A^{*}, A ; S\right) \cap L_{1}$ has $\left(S \cap L_{1}\right) \cdot\left(-\infty, T_{1}\right] \subseteq \operatorname{int}_{X}\left(N_{1}\right),\left(S \cap L_{1}\right) \cdot\left[T_{0}, \infty\right) \subseteq \operatorname{int}_{X}\left(N_{0}\right)$. Let $T=\frac{1}{2}\left(T_{0}-T_{1}\right)$, and replace $\left(N_{1}, L_{1}\right),\left(N_{0}, L_{0}\right)$ by $\left(N_{1}^{T}, L_{1}^{T}\right),\left(N_{0}^{T}, L_{0}^{T}\right)$, where $N^{T}=\bigcap_{-T \leq t \leq T} N \cdot t$ and $L^{T}=L \cdot(-T) \cap N^{T}$. Then $S \cap L_{1}^{T}$ is a section of $C\left(A^{*}, A ; S\right)$ with the property that every $x \in S \cap L_{1}^{T}$ has $x \cdot(-\infty, 0] \subseteq N_{1}, x \cdot(0, \infty) \cap N_{1}=\varnothing$. Since $C\left(A^{*}, A ; S\right)$ is open in $C\left(A^{*}, A ; X\right)$, there exists a compact neighborhood $U_{1}$ of $S \cap L_{1}^{T}$ in $L_{1}^{T}$ so that $U_{1} \cap C\left(A^{*}, A ; X\right)=S \cap L_{1}^{T} . U_{1}$ and $\varepsilon \geq 0$ may be chosen so that $M_{1}=N_{1} \cup U_{1} \cdot[0,4 T+\varepsilon] \cup N_{0}$ is a compact neighborhood of $S$ in $X$ with $U_{1} \cdot(0,4 T+\varepsilon] \cap N_{1}=\varnothing$. Similarly, in $\left(L_{0}^{-}\right)^{T}$ (the "entrance set" of $\left.N_{0}\right)$ there exists a compact neighborhood $U_{0}$ of $S \cap\left(L_{0}^{-}\right)^{T}$ such that $U_{0} \cap C\left(A^{*}, A ; X\right)=$ $S \cap\left(L_{0}^{-}\right)^{T}, U_{0} \cdot[-4 T-\varepsilon, 0) \cap N_{0}=\varnothing$, and $M_{0}=N_{1} \cup U_{0} \cdot[-4 T-\varepsilon, 0] \cup N_{0}$ is a compact neighborhood of $S$ in $X$.

Let $M=M_{1} \cap M_{0} . \quad M$ is a compact neighborhood of $S$. As $N_{1}$ and $N_{0}$ are disjoint compact sets, $I\left(N_{1} \cup N_{0}\right)=I\left(N_{1}\right) \cup I\left(N_{0}\right)=A^{*} \cup A$. If $x \in I(M) \backslash A^{*} \cup A$, then $x \cdot \mathbf{R}$ intersects $U_{1}$ and $U_{0}$, so there exist times $t_{1}<t_{0}$ such that $x \cdot\left(-\infty, t_{1}\right] \subseteq$ $N_{1}, x \cdot\left(t_{1}, t_{0}\right) \subseteq X \backslash\left(N_{1} \cup N_{0}\right), x \cdot\left[t_{0}, \infty\right) \subseteq N_{0}$. Thus $\omega^{*}(x) \subseteq A^{*}, \omega(x) \subseteq A$, and $x \in C\left(A^{*}, A ; X\right)$. That is, $I(M) \subseteq A^{*} \cup C\left(A^{*}, A ; X\right) \cup A$. Then by the choice of $U_{1}$ and $U_{0}, I(M)=S$.

COROLlaRY 2.2. Suppose $S$ is a compact invariant set in $X$.

(i) If $\left\{S_{i}\right\}$ is an invariant separation of $S$, then $S$ is isolated in $X$ if and only if each $S_{i}$ is. 
(ii) If $S$ is isolated with attractor-repeller pair $\left(A, A^{*}\right)$ and $\left\{C_{i}\right\}$ a separation of $C\left(A^{*}, A ; S\right)$, then each $S_{i}=A \cup C_{i} \cup A^{*}$ is isolated.

ProOF. (i) If $\left\{S_{i}\right\}$ is a separation of $S$ with each $S_{i}$ isolated in $X$, choose disjoint isolating neighborhoods $N_{i}$ for the $S_{i}$ in $X$. Then $N=\bigcup N_{i}$ is an isolating neighborhood for $S$ in $X$. If $S$ is isolated, choose an isolating neighborhood for $S$ in $X . S \backslash S_{i}$ and $S_{i}$ are closed in $S$, so they are closed in $N$. Choose disjoint open neighborhoods $U, V$ of $S_{i}$ and $S \backslash S_{i}$ in $\operatorname{int}_{X} N$. Then $\bar{U}$ is a compact neighborhood of $S_{i}$ in $X$ with $I(\bar{U})=S_{i}$.

(ii) If $S$ is isolated in $X$, then $A^{*}$ and $A$ are isolated in $X$ [1, III, 7.1], hence in $S$, and each $C_{i}$ is open in $C\left(A^{*}, A ; S\right)$. Thus $S_{i}$ is isolated in $S$ by 2.1 , and $S$ is isolated in $X$, so $S_{i}$ is isolated in $X$.

If $S$ is isolated with attractor-repeller pair $\left(A, A^{*}\right)$ and separation $\left\{C_{i}\right\}$ of $C\left(A^{*}, A ; S\right)$, then there exist exact sequences

$$
\cdots \rightarrow C H_{n}(X ; A) \rightarrow C H_{n}(X ; S) \rightarrow C H_{n}\left(X ; A^{*}\right) \rightarrow C H_{n-1}(X ; A) \rightarrow \cdots,
$$

the homology attractor-repeller sequence of $\left(S ; A, A^{*}\right)$, and

$$
\cdots \rightarrow C H_{n}(X ; A) \rightarrow C H_{n}\left(X ; S_{i}\right) \rightarrow C H_{n}\left(X ; A^{*}\right) \rightarrow C H_{n-1}(X ; A) \rightarrow \cdots,
$$

the homology attractor-repeller sequence of $\left(S_{i} ; A, A^{*}\right)$. The $\left(S ; A, A^{*}\right)$ sequence has connection map $\partial: C H_{n}\left(X ; A^{*}\right) \rightarrow C H_{n-1}(X ; A)$; the $\left(S_{i} ; A, A^{*}\right)$ sequence has connection map $\partial_{i}: C H_{n}\left(X ; A^{*}\right) \rightarrow C H_{n-1}(X ; A)$. To relate these maps, the following index triple constructions are required:

LEMMA 2.3. If $(X ; S) \in$ IS with $\left\{C_{i}\right\}$ a separation of $C\left(A^{*}, A ; S\right)$ and $S_{i}=$ $A \cup C_{i} \cup A^{*}$, there exists an isolating neighborhood for $(X ; S)$ of the form $\tilde{N}=$ $N \cup\left(\bigcup N_{i}\right) \cup N^{*}$, with $N, N_{i}, N^{*}$ compact such that

(i) $N$ is positively invariant, $N^{*}$ negatively invariant, relative to $\tilde{N}$.

(ii) $N \cap N^{*}=\varnothing, N_{i} \cap N_{j}=\varnothing$ for $i \neq j$, and $N_{i}$ intersects $N \cup N^{*}$ along their common boundaries.

(iii) $N$ is an isolating neighborhood for $A, N^{*}$ is an isolating neighborhood for $A^{*}$, and $\tilde{N}_{i}=N \cup N_{i} \cup N^{*}$ an isolating neighborhood for $S_{i}$.

PrOOF. Let $M$ be an isolating neighborhood for $S$ in $X$. Choose a regular index triple $\left(M_{2}, M_{1}, M_{0}\right)$ positively invariant relative to $M$. Then $M_{1}$ is an isolating neighborhood for $A$ and $\overline{M_{2} \backslash M_{1}}$ is an isolating neighborhood for $A^{*}$. There exists a $T>0$ so that no $x \in \partial_{M_{2}} M_{1}$ has $x \cdot[-T, T] \subseteq \partial_{M_{2}} M_{1}$. Take $N=\{x \in$ $\left.M_{1} \mid x \cdot[-T, 0] \cap \overline{M_{2} \backslash M_{1}}=\varnothing\right\}, N^{*}=\left\{x \in \overline{M_{2} \backslash M_{1}} \mid x \cdot[0, T] \cap M_{1}=\varnothing\right\} . \quad N$ and $N^{*}$ are then disjoint isolating neighborhoods for $A$ and $A^{*}$, positively (resp. negatively) invariant relative to $M_{2}$.

For $i \neq j$, let $r_{i j}=\min \left\{d(x, y) \mid x \in \overline{C_{i} \backslash\left(N \cup N^{*}\right)}, y \in \overline{C_{j} \backslash\left(N \cup N^{*}\right)}\right\}$ and let $r=\frac{1}{2} \min \left\{r_{i j}\right\}$. Let $N_{i}=\left\{x \in \overline{M_{2} \backslash\left(N \cup N^{*}\right)} \mid d\left(x, \overline{C_{i} \backslash\left(N \cup N^{*}\right)}\right) \leq r\right\}$. The $N_{i}$ are clearly compact, and satisfy (ii). For each $i, N \cup N_{i} \cup N^{*}$ is a neighborhood for $S_{i}$ which misses some element of $C_{j}$ for all $j \neq i$. Thus it is an isolating neighborhood for $S_{i}$. Further, $S=\bigcup S_{i} \subseteq \bigcup\left(N \cup N_{i} \cup N^{*}\right)=\tilde{N} \subseteq M_{2}$, so $\tilde{N}$ is an isolating neighborhood for $S$, and $N$ (resp. $N^{*}$ ) is positively (resp. negatively) invariant relative to $\tilde{N}$. 
LEMMA 2.4. In the notation of Lemma 2.3, if $(\bar{N}, \bar{L})$ is a (regular) index pair for $S$ in $X$ which is positively invariant in $\tilde{N}$, then $(\bar{N}, \bar{L} \cup(\bar{N} \cap N), \bar{L})$ and $\left(\bar{N}, \bar{L} \cup \operatorname{cl}_{X}\left(\bar{N} \backslash N^{*}\right), \bar{L}\right)$ are (regular) index triples for $\left(S ; A, A^{*}\right)$.

ProOF. For the triples to be index triples, the pairs $(\bar{L} \cup(\bar{N} \cap N), \bar{L})$ and $\left(\bar{L} \cup \operatorname{cl}_{X}\left(\bar{N} \backslash N^{*}\right), \bar{L}\right)$ must be index pairs for $A$ in $X$. But

$$
\operatorname{cl}_{X}((\bar{L} \cup(\bar{N} \cap N)) \backslash \bar{L})=\operatorname{cl}_{X}(\bar{N} \backslash \bar{L}) \cap N
$$

and

$$
\operatorname{cl}_{X}\left(\left(\bar{L} \cup \operatorname{cl}_{X}\left(\bar{N} \backslash N^{*}\right)\right) \backslash \bar{L}\right)=\operatorname{cl}_{X}\left(\bar{N} \backslash\left(\bar{L} \cup N^{*}\right)\right)
$$

are isolating neighborhoods of $A$; and $\bar{L}$ is a positively invariant exit set for $\bar{N}$, hence for $\bar{L} \cup(\bar{N} \cap N)$ and $\bar{L} \cup \operatorname{cl}_{X}\left(\bar{N} \backslash N^{*}\right)$.

The same argument shows that $\left(\bar{N} \cap \tilde{N}_{i},\left(\bar{L} \cap \tilde{N}_{i}\right) \cup(\bar{N} \cap N), \bar{L} \cap \tilde{N}_{i}\right)$ and $\left(\bar{N} \cap \tilde{N}_{i},\left(\bar{L} \cap \tilde{N}_{i}\right) \cup\left(\bar{N} \cap\left(N \cup N_{i}\right)\right), \bar{L} \cap \tilde{N}_{i}\right)$ are index triples for $\left(S_{i} ; A, A^{*}\right)$.

THEOREM 2.5. Suppose $S$ is an isolated invariant set with attractor-repeller pair $\left(A, A^{*}\right)$ and separation $\left\{C_{i}\right\}$ of $C\left(A^{*}, A ; S\right)$. If $\partial$ is the connection map of $\left(S ; A, A^{*}\right)$ and $\partial_{i}$ is the connection map of $\left(S_{i} ; A, A^{*}\right)$, then $\partial=\sum_{i=1}^{n} \partial_{i}$.

PROOF. It suffices to prove the theorem for $n=2$. Take a regular index triple $\left(\bar{N}_{2}, \bar{N}_{1}, \bar{N}_{0}\right)=(\bar{N}, \bar{L} \cup(\bar{N} \cap N), \bar{L})$ as in 2.4. Then $\bar{N}$ can be viewed as an identification of the spaces $N \cup N_{1} \cup N^{*}$ and $N \cup N_{2} \cup N^{*}$, formed by identifying the copies of $N$ and $N^{*}$. Form $M$ by identifying the $N \cup N_{1} \cup N^{*}$ and $N \cup N_{2} \cup N^{*}$ along $N^{*}$ only, and let $p: M \rightarrow \bar{N}_{2}$ be the natural projection map. Then the map of triples $\left(M_{2}, M_{1}, M_{0}\right)=\left(M, p^{-1}\left(\bar{N}_{1}\right), p^{-1}\left(\bar{N}_{0}\right)\right) \rightarrow\left(\bar{N}_{2}, \bar{N}_{1}, \bar{N}_{0}\right)$ induces a commutative diagram

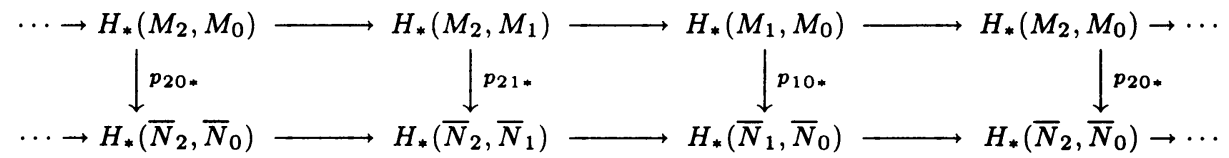

(i) $p_{21}:\left(M_{2}, M_{1}\right) \rightarrow\left(\bar{N}_{2}, \bar{N}_{1}\right)$ is a relative homeomorphism, so $p_{21 *}$ is an isomorphism.

(ii) By excision, there is a commutative diagram

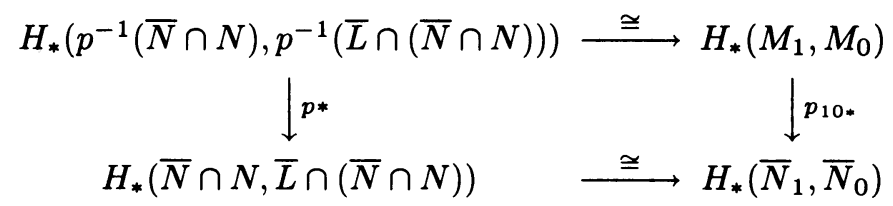

Over $N \cap \bar{N}, p$ is a disjoint double cover, so

$$
\begin{aligned}
& H_{*}\left(p^{-1}(\bar{N} \cap N), p^{-1}(\bar{L} \cap(\bar{N} \cap N))\right) \\
& \quad \cong H_{*}(\bar{N} \cap N, \bar{L} \cap(\bar{N} \cap N)) \oplus H_{*}(\bar{N} \cap N, \bar{L} \cap(\bar{N} \cap N)),
\end{aligned}
$$

with $p_{*}=\mathrm{id}_{*}+\mathrm{id}_{*}$.

(iii) As $\left(\bar{N}_{2}, \bar{N}_{1}, \bar{N}_{0}\right)$ is an index triple for $\left(S ; A, A^{*}\right), \partial_{N}$ is the connection map of $\left(S ; A, A^{*}\right)$. 
(iv) By excision, the components of the composition

$$
\begin{aligned}
& H_{*}\left(M_{2}, M_{1}\right) \stackrel{\partial_{M}}{\longrightarrow} H_{*}\left(p^{-1}(\bar{N} \cap N), p^{-1}(\bar{L} \cap(\bar{N} \cap N))\right) \\
& \quad \cong H_{*}(\bar{N} \cap N, \bar{L} \cap(\bar{N} \cap N)) \oplus H_{*}(\bar{N} \cap N, \bar{L} \cap(\bar{N} \cap N))
\end{aligned}
$$

are $\left(\partial_{1}, \partial_{2}\right)$. Namely, to compute one component, excise the other copy of $N \cap \bar{N}$ in $p^{-1}(N \cap \bar{N})$. The resulting triple is then an index triple for $\left(S_{i} ; A, A^{*}\right)$, so the map is $\partial_{i}$.

Then $\partial=\partial \circ p_{21 *}=p_{10 *} \circ \partial=\left(\mathrm{id}_{*}+\mathrm{id}_{*}\right) \circ\left(\partial_{1}, \partial_{2}\right)=\partial_{1}+\partial_{2}$.

COROLlaRY 2.6. Suppose $S$ is isolated with attractor-repeller pair $\left(A, A^{*}\right)$, $\left\{C_{i}\right\}$ a separation of $\bigcup C_{i} \subseteq C\left(A^{*}, A ; S\right)$ such that each $S_{i}=A \cup C_{i} \cup A^{*}$ is isolated. If $\partial \neq \sum_{i=1}^{n} \partial_{i}$, then $\bigcup C_{i} \neq C\left(A^{*}, A ; S\right)$.

In [9], Mischaikow studies homoclinic orbits in Hamiltonian systems by relating them to connecting orbits of an attractor-repeller pair in an associated gradient-like system. The connection map associated to each homoclinic is an isomorphism, but the connection map of the total set does not change when the associated heteroclinic orbit is attached. Corollary 2.6 shows that attaching the heteroclinic forces other connecting orbits to be created as well.

3. Hyperbolic critical point attractor-repeller pairs. The simplest example of a connection map is obtained by taking a $C^{2}$ flow on a manifold $M$. Suppose $S$ is an isolated invariant set with attractor-repeller pair $\left(\left\{x_{0}\right\},\left\{x_{1}\right\}\right), x_{1}$ and $x_{0}$ hyperbolic critical points of index $k_{0}$ and $k_{1}$ respectively. When $k_{1}=k_{0}+$ $1, C H_{*}\left(X ;\left\{x_{1}\right\}\right)$ consists of a single copy of $\mathbf{Z}$ in dimension $k_{0}+1 ; C H_{*}\left(X ;\left\{x_{0}\right\}\right)$ consists of a single copy of $\mathbf{Z}$ in dimension $k_{0}$. The connection map is then a homomorphism $\partial: \mathbf{Z} \rightarrow \mathbf{Z}$. We now compute this homomorphism in the case of transverse intersection of $W^{u}\left(x_{1}\right)$ and $W^{s}\left(x_{0}\right)$.

THEOREM 3.1. If $x_{1}, x_{0}$ are hyperbolic critical points in a manifold $M$ with indices $k+1$ and $k$ and $W^{u}\left(x_{1}\right)$ transverse to $W^{s}\left(x_{0}\right)$, then every connecting orbit $\gamma \in C\left(x_{1}, x_{0}\right)$ has $\bar{\gamma}=\left\{x_{1}\right\} \cup \gamma \cup\left\{x_{0}\right\}$ an isolated invariant set with $I(M ; \bar{\gamma})=\overline{0}$.

PROOF. As $x_{1}$ and $x_{0}$ are hyperbolic, they are isolated. $W^{u}\left(x_{1}\right)$ is transverse to $W^{s}\left(x_{0}\right)$, so $C\left(x_{1}, x_{0}\right)$ consists of a finite number of orbits. Thus $\gamma$ is a component of $C\left(x_{1}, x_{0}\right)$ and $\bar{\gamma}$ is isolated.

To show $I(M ; \bar{\gamma})=\overline{0}$, consider first $M=\mathbf{R}^{n}$ with a flow such that $x_{1}=$ $(-1,0, \ldots, 0)$ and $x_{0}=(1,0, \ldots, 0)$ are hyperbolic critical points of index $k+1$ and $k$ respectively, $\bar{\gamma}_{0}=\{(x, 0, \ldots, 0)|| x \mid \leq 1\}$, and the $x_{1}$-axis is invariant under the flow. We show $I\left(\mathbf{R}^{n} ; \bar{\gamma}_{0}\right)=\overline{0}$ by a series of continuations. Write $\mathbf{x} \in \mathbf{R}^{n}$ as $(x, \mathbf{y})$, with $\mathbf{y} \in\{0\} \times \mathbf{R}^{n-1}$, and suppose the flow is given by vectorfield $X(\mathbf{x})$.

By the rescaling $(t, x, \mathbf{y}) \mapsto\left(t, x, \varepsilon^{-1} \mathbf{y}\right), X$ can be perturbed to its first order (in y) terms. $\bar{\gamma}_{0}$ is unchanged by this rescaling, and continues throughout the rescaling. That is, $\bar{\gamma}_{0}$ in the $X$ flow is related by continuation to $\bar{\gamma}_{0}$ in the flow induced by $X_{1}(x, \mathbf{y})=\left(X(x, \mathbf{0})+X_{1}(x) \mathbf{y}, X_{2}(x) \mathbf{y}, \ldots, X_{n}(x) \mathbf{y}\right)$. Next, continue to $X_{2}(x, \mathbf{y})=\left(X(x, \mathbf{0}), X_{2}(x) \mathbf{y}, \ldots, X_{n}(x) \mathbf{y}\right)$. For every $x, \mathbf{0}$ is an isolated rest point in the $A(x) \mathbf{y}=\left(X_{2}(x) \mathbf{y}, \ldots, X_{n}(x) \mathbf{y}\right)$ flow on $\mathbf{R}^{n-1}$ with $I\left(\mathbf{R}^{n-1} ;\{\mathbf{0}\}\right)=\Sigma^{k}$. As $\bar{\gamma}_{0}$ is isolated in the $X(x, \mathbf{0})$ flow on $\mathbf{R}, \bar{\gamma}_{0}=\bar{\gamma}_{0} \times\{\mathbf{0}\}$ is isolated in the $X_{2}$ flow, and related by continuation to $\bar{\gamma}_{0}$ in the $X_{1}$ flow. The $X_{2}$ flow continues to a product 
flow $X_{3}(x, \mathbf{y})=(X(x, \mathbf{y}), A(0) \mathbf{y})$, with $\bar{\gamma}_{0}$ continuing throughout. Then in the $X_{3}$ flow, $I\left(\mathbf{R}^{n} ; \bar{\gamma}_{0}\right)=I\left(\mathbf{R} ; \bar{\gamma}_{0}\right) \times I\left(\mathbf{R}^{n-1} ;\{\mathbf{0}\}\right)$, with $I\left(\mathbf{R} ; \bar{\gamma}_{0}\right)=\overline{0}$. That is, $\bar{\gamma}_{0}$ has an isolating neighborhood $\{|t| \leq 1+\varepsilon\}$, with exit set $\{-1-\varepsilon\}$.

For the general case, choose a map $f: \gamma \rightarrow \mathbf{R}^{n}$ taking $\gamma$ diffeomorphically onto $\gamma_{0}=(-1,1) \times \mathbf{R}^{n-1}$. Choose orbits $\gamma_{u} \in W^{u}\left(x_{1}\right), \gamma_{s} \in W^{s}\left(x_{0}\right)$ whose unit tangent vectors at $x_{1}, x_{0}$ are negatives of those of $\gamma$, and a smooth embedding

$$
f: \Gamma=\gamma_{u} \cup\left\{x_{1}\right\} \cup \gamma \cup\left\{x_{0}\right\} \cup \gamma_{s} \rightarrow \mathbf{R} \times\{\mathbf{0}\} \subseteq \mathbf{R}^{n} .
$$

(The flow can be perturbed away from $\gamma$ if necessary to guarantee that $f$ is an embedding.) As $\Gamma \cong \mathbf{R}, f$ extends to a tubular neighborhood $f: E \rightarrow \mathbf{R}^{n}$. The flow $\dot{\mathbf{x}}=D f\left(f^{-1}(\mathbf{x})\right) X\left(f^{-1}(\mathbf{x})\right)$ on $\mathbf{R}^{n}$ has $I\left(\mathbf{R}^{n} ; \bar{\gamma}_{0}\right)=\overline{0}$, and $f$ is a flow map. Thus $f^{-1}\left(\bar{\gamma}_{0}\right)=\bar{\gamma}$ is isolated in $E$ (hence in $\left.M\right)$ and $I(f)$ is an isomorphism, so $I(M ; \bar{\gamma})=I(E ; \bar{\gamma})=I\left(\mathbf{R}^{n} ; \bar{\gamma}_{0}\right)=\overline{0}$.

COROLLARY 3.2. For each such $\gamma,\left(\left\{x_{1}\right\},\left\{x_{0}\right\}\right)$ is an attractor-repeller pair for $\bar{\gamma}$, with connection map $\partial: C H_{k+1}\left(M ; x_{1}\right) \rightarrow C H_{k}\left(M ; x_{0}\right)$ an isomorphism.

COROLLARY 3.3. If $k_{1}=k_{0}+1$ and $W^{u}\left(x_{1}\right)$ is transverse to $W^{s}\left(x_{0}\right)$, then $S=\operatorname{cl}_{M} C\left(x_{1}, x_{0}\right)$ is an isolated invariant set. $C\left(x_{1}, x_{0}\right)$ consists of finitely many orbits, with $\left|C\left(x_{1}, x_{0}\right) / \mathbf{R}\right| \geq|\partial|$ and $\left|C\left(x_{1}, x_{0}\right) / \mathbf{R}\right| \equiv|\partial| \bmod 2$, where $|\partial|=n$ if and only if $\operatorname{coker}(\partial) \cong \mathbf{Z} / n \mathbf{Z}$.

ProOF. By transversality, $C\left(x_{1}, x_{0}\right) / \mathbf{R}$ is finite and the collection of connecting orbits is an invariant separation of $C\left(x_{1}, x_{0}\right)$. For each orbit, the connection map is an isomorphism, so coker $\left(\partial_{i}\right)=0$ and $\left|\partial_{i}\right|=1$. As $\partial=\sum_{i=1}^{n} \partial_{i}$,

$$
|\partial| \leq \sum_{i=1}^{n}\left|\partial_{i}\right|=\left|C\left(x_{1}, x_{0}\right) / \mathbf{R}\right|, \quad \text { and } \quad|\partial| \equiv \partial \equiv \sum_{i=1}^{n} \partial_{i} \equiv \sum_{i=1}^{n}\left|\partial_{i}\right| \bmod 2 \text {. }
$$

Thus, in the case of transverse intersection, $C\left(x_{1}, x_{0} ; S\right)$ has a finest invariant separation (with elements consisting of single connecting orbits) and $\partial_{S}$ is trivial only when the isomorphisms $\partial_{i}$ of the orbits cancel. If $W^{u}\left(x_{1}\right)$ and $W^{s}\left(x_{0}\right)$ are not transverse, there exists isolated $\left(S ; x_{0}, x_{1}\right)$ such that $C\left(x_{1}, x_{0} ; S\right)$ is connected and $\partial_{S}$ is trivial. However, if $W^{u}\left(x_{1}\right)$ and $W^{s}\left(x_{0}\right)$ are not transverse, they can be made transverse by an arbitrarily small perturbation of the flow. For sufficiently small perturbations, $\left(S ; x_{0}, S_{1}\right)$ continues as an attractor-repeller decomposition, so $\partial_{S}$ remains unchanged. That is, every transverse perturbation has connecting orbit set $C\left(x_{1}, x_{0} ; S^{\prime}\right)$ with $\left|C\left(x_{1}, x_{0} ; S^{\prime}\right) / \mathbf{R}\right| \geq\left|\partial_{S}\right|$.

We now return to the flows on spheres from $\S 1$ in the following setting: $M$ a manifold with $C^{2}$ flow, $x_{0}, x_{1}$ hyperbolic critical points of index 0 and $k, S$ an isolated invariant set with $\left(\left\{x_{0}\right\},\left\{x_{1}\right\}\right)$ an attractor-repeller pair. In this setting, $W^{u}\left(x_{1}\right)$ and $W^{s}\left(x_{0}\right)$ are necessarily transverse.

THEOREM 3.4. Suppose $S$ is a compact invariant set with attractor-repeller pair $\left(\left\{x_{0}\right\},\left\{x_{1}\right\}\right)$. Then $S$ is isolated in $M$ if and only if $S$ is a topological embedding of a 0 -sphere or a $k$-sphere, or $k=1$ and $S$ is a topological embedding of the unit interval. If $S$ is a sphere, the connection map of $\left(S ;\left\{x_{1}\right\},\left\{x_{0}\right\}\right)$ is trivial; if $S$ is an interval, the connection map of $\left(S ;\left\{x_{1}\right\},\left\{x_{0}\right\}\right)$ is an isomorphism.

Proof. (i) Suppose $S$ is an embedding of $S^{0}, S^{k}$, or an interval. For $k=1$, it follows from 3.1 that $S$ is isolated in $M$. For $k>1$, we apply 2.1. As $x_{1}$ 
and $x_{0}$ are hyperbolic, they are isolated. Embeddings of spheres are compact, so $S$ will be isolated if $C\left(x_{1}, x_{0} ; S\right)$ is open in $C\left(x_{1}, x_{0}\right)$. If $S$ is an embedding of $S^{0}, C\left(x_{1}, x_{0} ; S\right)=\varnothing$; if $S$ is an embedding of $S^{k}, C\left(x_{1}, x_{0} ; S\right)=C\left(x_{1}, x_{0}\right)$.

(ii) Suppose $S$ is isolated and $k=1$. $W^{u}\left(x_{1}\right)$ consists of $x_{1}$ and two orbits unstable to $x_{1}$. Thus $C\left(x_{1}, x_{0} ; S\right)$ consists of 0,1 or 2 orbits. The intersection $W^{u}\left(x_{1}\right) \cap W^{s}\left(x_{0}\right)$ is necessarily transverse, so $S$ is a topological embedding of $S^{0},[0,1]$, or $S^{1}$.

The relevant segment of the homology attractor-repeller sequence is

$$
0 \rightarrow C H_{1}(M ; S) \rightarrow C H_{1}\left(M ; x_{1}\right) \stackrel{\partial}{\rightarrow} C H_{0}\left(M ; x_{0}\right) \rightarrow C H_{0}(M ; S) \rightarrow 0 .
$$

$C H_{0}(M ; S) \cong \tilde{H}_{0}(N / L)$ for some index pair $(N, L)$. In particular, it is free abelian. As $C H_{0}\left(M ; x_{0}\right) \cong \mathbf{Z}$ maps onto it, it is either 0 or $\mathbf{Z}$. From the exact sequence, $C H_{0}(M ; S)=\operatorname{coker}(\partial)$, so by $3.3,|\partial| \leq 1$.

If $S \cong S^{0}$, then $C\left(x_{1}, x_{0}\right)=\varnothing$ and $\partial=0$.

If $S \cong[0,1]$, then $\partial$ is an isomorphism by 3.2 .

If $S \cong S^{1}$, then by deleting the connecting orbits one at a time, isolated invariant sets $S_{1}, S_{2}$ are formed with $\partial=\partial_{1}+\partial_{2}$ and $\partial_{1}$ and $\partial_{2}$ isomorphisms. Thus $|\partial|=0,2$. But $|\partial| \leq 1$, so $\partial=|\partial|=0$.

(iii) If $S$ is isolated and $k>1$, dimensions force the connection map to be trivial. If $C\left(x_{1}, x_{0} ; S\right)=\varnothing$, then $S$ is a 0 -sphere. If $C\left(x_{1}, x_{0} ; S\right) \neq \varnothing$, ther $W^{\iota}\left(x_{1}\right)=\left\{x_{1}\right\} \cup C\left(x_{1}, x_{0} ; S\right)$.

That is, $\left\{x_{1}\right\} \cup C\left(x_{1}, x_{0} ; S\right) \subseteq W^{u}\left(x_{1}\right)$ by definition. Suppose

$$
W^{u}\left(x_{1}\right) \not \subset\left\{x_{1}\right\} \cup C\left(x_{1}, x_{0} ; S\right) \text {. }
$$

If $C\left(x_{1}, x_{0} ; S\right) \neq \varnothing$, choose a $y_{1} \in C\left(x_{1}, x_{0} ; S\right)$ and a $y_{2} \in W^{u}\left(x_{1}\right) \backslash C\left(x_{1}, x_{0} ; S\right)$. Let $M_{0}=\operatorname{cl}_{M}\left(\left\{x_{0}, x_{1}, y_{1}, y_{2}\right\} \cdot \mathbf{R}\right) . \quad M_{0}$ is a closed invariant subset of $M$ with $M_{0} \cap S=\left\{x_{0}, x_{1}, y_{1}\right\} \cdot \mathbf{R}$. In $M_{0}, x_{1}$ and $x_{0}$ are hyperbolic with indices 1 and 0 and $y_{1} \cdot \mathbf{R}$ is the sole connecting orbit. Then from (ii), the homology indices are

$$
\begin{aligned}
C H_{*}\left(M_{0} ; x_{0}\right) & =(\mathbf{Z}, 0,0, \ldots), \\
C H_{*}\left(M_{0} ; M_{0} \cap S\right) & =(0,0,0, \ldots), \\
C H_{*}\left(M_{0} ; x_{1}\right) & =(0, \mathbf{Z}, 0, \ldots),
\end{aligned}
$$

and the $M_{0}$ connection map $\partial_{0}: C H_{1}\left(M_{0} ; x_{1}\right) \rightarrow C H_{0}\left(M_{0} ; x_{0}\right)$ is an isomorphism.

But $C H_{*}\left(M ; x_{0}\right)=(\mathbf{Z}, 0,0, \ldots), C H_{*}\left(M ; x_{1}\right)=(0, \ldots, 0, \mathbf{Z}, 0, \ldots)$ (with the $\mathbf{Z}$ in dimension $k>1$ ), so it must have $\partial_{M}=0$. Further, the inclusion $i: M_{0} \rightarrow M$ must have $i_{*}: C H_{*}\left(M_{0} ; x_{1}\right) \rightarrow C H_{*}\left(M ; x_{1}\right)$ trivial and

$$
i_{*}: C H_{*}\left(M_{0} ; x_{0}\right) \rightarrow C H_{*}\left(M ; x_{0}\right)
$$

an isomorphism. That is, $i_{*} \circ \partial_{0}$ is an isomorphism and $\partial_{M} \circ i_{*}$ is trivial, so $i_{*} \circ \partial_{0} \neq \partial_{M} \circ i_{*}$. But in [7] it is shown that $i_{*} \circ \partial_{0}=\partial_{M} \circ i_{*}$, so $W^{u}\left(x_{1}\right) \subseteq$ $\left\{x_{1}\right\} \cup C\left(x_{1}, x_{0} ; S\right)$.

Thus $\left\{x_{1}\right\} \cup C\left(x_{1}, x_{0} ; S\right)$ is an injective immersion of $\mathbf{R}^{k}$ in $M$ by the stable manifold theorem. As $C\left(x_{1}, x_{0} ; S\right) \subseteq W^{s}\left(x_{0}\right)$, this extends to a continuous bijection of $S^{k}$ onto $S$ by sending $\infty$ to $x_{0} . S^{k}$ is compact and $M$ is Hausdorff, so $S$ is a topological embedding of $S^{k}$ in $M$.

REMARKS. (i) This result differs from Reeb's theorem in that Reeb's hypothesis that $S$ be a compact manifold is replaced by the assumptions that $S$ be isolated in a manifold and have $k_{0}=0$ and $\partial$ trivial. 
(ii) $S$ need not be a submanifold of $M$, as the embedding of $W^{u}\left(x_{1}\right)$ need not extend differentiably to $x_{0}$.

(iii) The homology Conley index by itself is not sufficiently refined to distinguish the 0 -sphere and $k$-sphere cases. However, by considering additional structures on the index (such as the mappings introduced in [6]) it may be possible to distinguish them by some homological invariant.

\section{REFERENCES}

1. C. Conley, Isolated invariant sets and the Morse index, CBMS Regional Conf. Ser. in Math. no. 38, Amer. Math. Soc., Providence, R.I., 1978.

2. R. Franzosa, Index filtrations and the homology index braid for partially ordered Morse decompositions, Trans. Amer. Math. Soc. 298 (1986), 193-213.

3. _ The connection matrix theory for the Conley index, Trans. Amer. Math. Soc. (to appear).

4. H. Kurland, Homotopy invariants of repeller-attractor pairs. I. The Púppe sequence of an $R-A$ pair, J. Differential Equations 46 (1982), 1-31.

5. __ Isolated connections and chain recurrence, preprint.

6. C. McCord, Mappings and homological properties in the Conley index theory, Ergodic Theory Dynamical Systems (to appear).

7. L_ Mappings and Morse decompositions in the Conley index theory, preprint.

8. K. Mischaikow, Classification of traveling wave solutions of reaction-diffusion systems, Lefschetz Center for Dynamical Systems, no. 86-5, 1985.

9. __ Homoclinic orbits in Hamiltonian systems and heteroclinic orbits in gradient and gradientlike systems, Lefschetz Center for Dynamical Systems, no 86-33, 1986.

10. J. Reineck, Connecting orbits in one parameter families of flows, Ergodic Theory Dynamical Systems (to appear).

11. D. Salamon, Connected simple systems and the Conley index of isolated invariant sets, Trans. Amer. Math. Soc. 291 (1985), 1-41.

Department of Mathematical Sciences, University of Cincinnati, CincinNATI, OHIO 45221 